\title{
Research Article \\ Horizon Wavefunction of Generalized Uncertainty Principle Black Holes
}

\author{
Luciano Manfredi and Jonas Mureika \\ Department of Physics, Loyola Marymount University, Los Angeles, CA 90045-2659, USA \\ Correspondence should be addressed to Jonas Mureika; jmureika@lmu.edu
}

Received 27 September 2016; Revised 14 November 2016; Accepted 17 November 2016

Academic Editor: Elias C. Vagenas

Copyright ( 2016 L. Manfredi and J. Mureika. This is an open access article distributed under the Creative Commons Attribution License, which permits unrestricted use, distribution, and reproduction in any medium, provided the original work is properly cited. The publication of this article was funded by $\mathrm{SCOAP}^{3}$.

\begin{abstract}
We study the Horizon Wavefunction (HWF) description of a Generalized Uncertainty Principle inspired metric that admits subPlanckian black holes, where the black hole mass $m$ is replaced by $M=m\left(1+(\beta / 2)\left(M_{\mathrm{Pl}}^{2} / m^{2}\right)\right)$. Considering the case of a wave-packet shaped by a Gaussian distribution, we compute the HWF and the probability $\mathscr{P}_{\mathrm{BH}}$ that the source is a (quantum) black hole, that is, that it lies within its horizon radius. The case $\beta<0$ is qualitatively similar to the standard Schwarzschild case, and the general shape of $\mathscr{P}_{\mathrm{BH}}$ is maintained when decreasing the free parameter but shifted to reduce the probability for the particle to be a black hole accordingly. The probability grows with increasing mass slowly for more negative $\beta$ and drops to 0 for a minimum mass value. The scenario differs significantly for increasing $\beta>0$, where a minimum in $\mathscr{P}_{\mathrm{BH}}$ is encountered, thus meaning that every particle has some probability of decaying to a black hole. Furthermore, for sufficiently large $\beta$ we find that every particle is a quantum black hole, in agreement with the intuitive effect of increasing $\beta$, which creates larger $M$ and $R_{H}$ terms. This is likely due to a "dimensional reduction" feature of the model, where the black hole characteristics for sub-Planckian black holes mimic those in $(1+1)$ dimensions and the horizon size grows as $R_{H} \sim M^{-1}$.
\end{abstract}

\section{Introduction}

Black holes are special objects in gravitational physics because they are expected to reveal features of both classical and quantum gravitation. Indeed, one can note this connection in the simple fact that the defining parameter of the quantum gravity scale, the Planck mass $M_{\mathrm{Pl}}$, simultaneously sets the strength of classical gravitation $G=M_{\mathrm{Pl}}^{-2}$. A complete understanding of black hole physics will thus help shed light on this elusive theory. Although large black holes may reveal hints of quantum effects through, for example, the morphology of their shadows [1], it is anticipated that eventual observation of quantum scale black holes formed in high-energy collisions will provide direct evidence. In this regime, these objects transcend classical and quantum gravitation, and thus forming reliable predictions of their physics becomes tenuous in the absence of a complete theory of quantum gravity.

Although such a formulation is still incomplete, the literature is replete with first steps beyond the classical regime and into the quantum realm. Such semiclassical approaches generally rely on a classical framework, extended to include quantum effects at the appropriate energy or length scale that tame or remove the singularity. Examples include noncommutative geometry inspired models ([2]; also see [3] for an overview and additional references therein), the Generalized Uncertainty Principle [4, 5], and asymptotic safety [6]. Other approaches stem from quantum mechanical first principles and introduce gravitation as an energy or potential constraint. The Schrodinger-Newton equation $[7,8]$ can be derived from the weak-field Einstein's equations with the stress-energy tensor replaced by the expectation of a quantum operator, from which potential table-top quantum gravity measurements may be possible [9]. General aspects of quantum field theories in curved space-time are also well-known modifications of quantum theories to include gravitation [10-12].

A more recent approach in understanding the nature of quantum black holes is to consider the quantum mechanical 
conditions for their creation in terms of a wavefunction description. Using a framework known as the "Horizon Wavefunction" (HWF), the black hole is treated as a quantum particle whose spatial wavefunction is contained within its classical horizon radius [13, 14]. If such particles are created in high-energy collisions, then the chance of creating a black hole can be assessed by evaluating the associated probability. Particularly, the HWF has been used to understand aspects of quantum black hole thermodynamics, including evaporation signatures in four-dimensional space-time [15-19], as well as extra- and $(1+1)$-dimensional scenarios [20]. Potential experimentally detectable signatures that arise from such a description have been discussed in [21].

A particularly interesting feature of the HWF formalism is the appearance of a Generalized Uncertainty Principle (GUP), in which quantum uncertainties are simultaneously influenced by the wave and gravitational length scales of a particle. Originally noted as a feature of string theory [22], the GUP has been shown to be a largely model-independent prediction of quantum gravity theories, including loop quantum gravity [23], noncommutative quantum mechanics [24], gravity ultraviolet self-completeness [25], and other minimum length scenarios $[26,27]$. While most minimal length approaches yield a lower bound to black hole masses, a recently derived GUP-modified Schwarzschild metric was shown to allow the existence of sub-Planckian black holes [4]. A special feature of these sub-Planckian black holes is that their physical and thermodynamic characteristics mimic those of $(1+1)$-dimensional black holes-that is, the horizon size varies inversely with the black hole mass, $R_{H} \sim M_{\mathrm{BH}}^{-1}$, and the Hawking temperature linear in the mass, $T_{H} \sim M$.

Since the HWF can predict both the probability of black hole formation for arbitrary masses and also source constraints on a GUP from the quantum mechanical side, we seek to understand how encoding the GUP in the metric will influence the probability of black hole formation. In this paper, we apply the HWF prescription to the GUP-inspired metric of [4]. After first reviewing the formalism for both HWF and the GUP metric in Sections 2 and 3, we derive expressions for the HWF and black hole probabilities in both the super- and sub-Planckian mass regimes for varying GUP model parameters. In the former case, we find the results to be in agreement with those of the Schwarzschild HWF. In the sub-Planckian regime, we show that the probability of a particle of arbitrarily small mass becoming a black hole tends to unity. We discuss the results in the Conclusions.

\section{The Horizon Wavefunction Formalism}

In order to follow the prescription outlined above, we review the first ingredient of the approach, namely, the HWF framework. The following arguments reproduce the standard approach detailed in, for example, [13] and similar references. We start from the definition of the trapping surface:

$$
g^{i j} \nabla_{i} r \nabla_{j} r=0,
$$

where $\nabla_{i} r$ is normal to spherical surfaces of area $\mathscr{A}=4 \pi r^{2}$. From this, one can derive the metric function

$$
g^{r r}=1-\frac{2 \ell_{p}\left(m / M_{\mathrm{Pl}}\right)}{r},
$$

if one assigns coordinates $\left(x^{1}, x^{2}\right)=(t, r)$. The quantities $M_{\mathrm{Pl}}$ and $\ell_{p}$ are the Planck mass and length, respectively. Assuming a rough flat space, the Misner-Sharp mass can be calculated as

$$
m(r, t)=4 \pi \int_{0}^{r} \rho(\bar{r}, t) \bar{r}^{2} d \bar{r},
$$

where $\rho=\rho(r, t)$ is the local matter density. The condition for a trapping surface to be formed follows from the constraint that the gravitational radius is

$$
R_{S}(r, t) \geq r
$$

for a given value of coordinates $(t, r)$. If the source is completely contained within this region, then $R_{S}$ is identified with the usual Schwarzschild radius. More generally, condition (4) gives a more rigorous representation of the hoop conjecture [28], which allows for the formation of a black hole in the collision of two masses if their impact parameter $b$ is contained within the Schwarzschild radius. From the above definitions, this can be reexpressed as the condition

$$
b \lesssim \frac{2 \ell_{p} E}{M_{\mathrm{Pl}}} \equiv r_{H},
$$

where $E$ is the total energy in the centre-of-mass frame.

Since such an object would be manifestly quantum mechanical, one must also introduce an uncertainty in its position. This will be on the order of the system's Compton wavelength, $\lambda_{m} \simeq \ell_{p} M_{\mathrm{Pl}} / m$, providing the additional constraint on the gravitational radius

$$
\begin{aligned}
R_{S} & \gtrsim \lambda_{m} \Longrightarrow \\
m & \gtrsim M_{\mathrm{Pl}} .
\end{aligned}
$$

This spread in localization can be represented by the wavefunction

$$
\left|\psi_{S}\right\rangle=\sum_{E} C(E)\left|\psi_{E}\right\rangle
$$

As usual, the sum over the variable $E$ represents the decomposition on the spectrum of the Hamiltonian:

$$
\widehat{H}\left|\psi_{E}\right\rangle=E\left|\psi_{E}\right\rangle .
$$

Once the energy spectrum is known, we can use (5) to get

$$
E=M_{\mathrm{Pl}} \frac{r_{H}}{2 \ell_{p}} .
$$

One can now define the HWF as

$$
\psi_{H}\left(r_{H}\right)=C\left(\frac{M_{\mathrm{Pl}} r_{H}}{2 \ell_{p}}\right),
$$


which can be normalized as

$$
\left\langle\psi_{H} \mid \phi_{H}\right\rangle=4 \pi \int_{0}^{\infty} \psi_{H}^{*}\left(r_{H}\right) \phi_{H}\left(r_{H}\right) r_{H}^{2} d r_{H}
$$

Conceptually, the normalized HWF $\psi_{H}$ yields the probability for an observer to measure particle in the quantum state $\psi_{S}$ and associate with it a horizon of radius $r=r_{H}$. Consequently, the sharply defined classical radius is replaced by the expectation radius of the operator $\widehat{r}_{H}$.

The probability for the source to be a black hole is that it lies completely within its horizon:

$$
\mathscr{P}_{\mathrm{BH}}=\int_{0}^{\infty} \mathscr{P}_{<}\left(r<r_{H}\right) d r_{H},
$$

where the density

$$
\mathscr{P}_{<}\left(r<r_{H}\right)=\mathscr{P}_{S}\left(r<r_{H}\right) \mathscr{P}_{H}\left(r_{H}\right)
$$

is a combination of requiring the particle to rest within a sphere of radius $r=r_{H}$ and the probability that $r_{H}$ is the gravitational radius. These are, respectively, calculated as

$$
\begin{aligned}
\mathscr{P}_{S}\left(r<r_{H}\right) & =\int_{0}^{r_{H}} \mathscr{P}_{S}(r) d r=4 \pi \int_{0}^{r_{H}}\left|\psi_{S}(r)\right|^{2} r^{2} d r, \\
\mathscr{P}_{H}\left(r_{H}\right) & =4 \pi r_{H}^{2}\left|\psi_{H}\left(r_{H}\right)\right|^{2} .
\end{aligned}
$$

\section{Generalized Uncertainty Principle Black Holes}

As one approaches the Planck scale, it has been argued [22, 29-31] that the Heisenberg Uncertainty Principle (HUP) should be replaced by a Generalized Uncertainty Principle (GUP) of the form

$$
\Delta x>\frac{\hbar}{\Delta p}+\left(\frac{\alpha \ell_{p}^{2}}{\hbar}\right) \Delta p
$$

where $\alpha$ is a dimensionless constant that depends on the particular model of interest. This introduces a duality in the momentum uncertainty of the form $\Delta x \sim \Delta p+1 / \Delta p$, and, assuming the correspondence $\Delta p \rightarrow m$, one can determine a similar mass duality to be present in the characteristic length scale of the system.

The Planck scale is generally regarded as the transition point between classical and quantum regimes. Rewriting (15) using the substitution $\Delta x \rightarrow R$ and $\Delta p \rightarrow M$, one can define both a generalized Compton radius (approaching from the sub-Planckian regime) and a gravitational radius (approaching from the super-Planckian regime) [4]:

$$
\begin{aligned}
& R>R_{C}^{\prime}=\frac{\hbar}{M}+\alpha G M=\frac{\hbar}{M}\left[1+\alpha\left(\frac{M}{M_{\mathrm{Pl}}}\right)^{2}\right], \\
& R>R_{S}^{\prime}=\alpha G M\left[1+\frac{1}{\alpha}\left(\frac{M_{\mathrm{Pl}}}{M}\right)^{2}\right] .
\end{aligned}
$$

Note the right-hand side of the inequality in (17) becomes a GUP perturbation to the Schwarzschild radius if $\alpha=2$. There is no reason that $\alpha$ should possess such a generic constraint, however. Thus, the above expression is further generalized to a new parameter $\beta \neq \alpha$, giving

$$
R>R_{S}^{\prime}=2 G M\left[1+\frac{\beta}{2}\left(\frac{M_{\mathrm{Pl}}}{M}\right)^{2}\right] .
$$

The latter expression is the true GUP-modified horizon scale. The crossover of solutions to (16) and (18) can potentially allow for the simultaneous classification of particles as black holes. In fact, the expressions for $R_{C}^{\prime}$ and $R_{S}^{\prime}$ coincide when

$$
M_{f}=\sqrt{\frac{1-\beta}{2-\alpha}} M_{\mathrm{Pl}},
$$

where $M_{f}$ is the true fundamental mass scale at which the transition between quantum mechanics and classical gravity takes place. Proper choice of $\alpha$ and $\beta$ can thus keep particles and black holes separate. In this paper we will not deal any further with $\alpha$ or the generalized Compton scale; the interested reader is referred to a more detailed discussion in [4].

Although this characteristic was not derived from a general relativistic approach, one can posit that it should be. That is, the GUP itself is encoded in the space-time geometry and, as argued in [4], a metric description of spacetime must incorporate such a mass duality. In the large mass limit $M \gg M_{\mathrm{Pl}}$, where quantum effects are negligible, one should recover the Schwarzschild solution. In this case, the black hole mass is well-defined in terms of the stress-energy tensor. When $M<M_{\mathrm{Pl}}$, however, the exact meaning of the mass parameter becomes ambiguous, referring to both a particle and a black hole. Since the horizon radius of subPlanckian mass black holes would be shorter than the Planck length itself, the relativistic description becomes unreliable. Consequently, one takes the object to be a particle of mass $M \sim \hbar / \lambda_{C}$, with $\lambda_{C}$ being the Compton wavelength. This can also be expressed as a variant of the Komar mass:

$$
M \equiv \int_{\Sigma} d^{3} x \sqrt{\gamma} n_{\mu} K_{\nu} T^{\mu \nu} \simeq-4 \pi \int_{0}^{\lambda_{C}} d r r^{2} T_{0}^{0},
$$

where $\gamma$ is the determinant of the spatially induced metric $\gamma^{i j}$, $n_{\mu}$ is a time-like unit vector, $K_{\nu}$ is a killing vector describing time-translation symmetry, $T^{\mu \nu}$ is the stress-energy tensor, and $T_{0}^{0}$ quantifies the energy density over the length scale $\lambda_{C}$.

Since we lack a full quantum theory of gravity, however, the exact form of the stress-energy tensor above is nebulous, and so one can assume the value of $T_{0}^{0}$ to represent some quantum mechanical distribution of matter [4]. Consequently, the full definition of the mass will include both the large scale (e.g., ADM) mass and the short scale particle mass.

Incorporating this new relation for the mass, one arrives at a quantum corrected form of the Schwarzschild metric [4]:

$$
\begin{aligned}
& d s^{2}=F(r) d t^{2}-F(r)^{-1} d r^{2}-r^{2} d \Omega^{2}, \\
& F(r)=1-\frac{2}{M_{\mathrm{Pl}}^{2}} \frac{M}{r}\left(1+\frac{\beta}{2} \frac{M_{\mathrm{Pl}}^{2}}{M^{2}}\right) .
\end{aligned}
$$




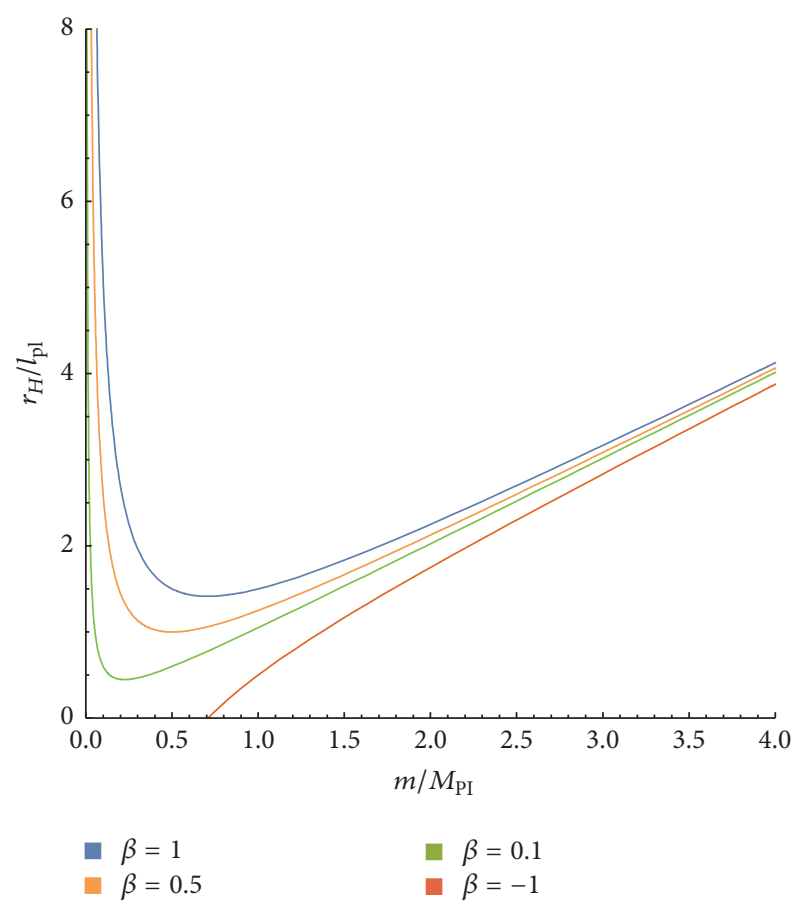

FIGURE 1: Horizon radius (22) as a function of mass $M / M_{\mathrm{Pl}}$ for $\beta=1$ (blue, top), $\beta=0.5$ (orange, second from top), $\beta=0.1$ (green, third from top), and $\beta=-1$ (red, bottom). In the last case, the horizon vanishes when $M=\sqrt{|\beta| / 2} M_{\mathrm{Pl}}$ and is defined only for bigger masses than this cut-off. This Figure is reproduced from [4].

In essence, this metric encapsulates all features of the Schwarzschild solution by virtue of the fact that the modification in the mass term is coordinate-independent. Furthermore, natural dimensional reduction features are demonstrated in the gravitational radius and thermodynamics of sub-Planckian objects $\left(m \ll M_{\mathrm{Pl}}\right)$ that resemble that of $(1+1)$-D gravity.

Specifically, the horizon is

$$
r_{H}=\frac{2}{M_{\mathrm{Pl}}^{2}}\left(\frac{M^{2}+(\beta / 2) M_{\mathrm{Pl}}^{2}}{M}\right)
$$

which yields

$$
\begin{aligned}
& M \gg M_{\mathrm{Pl}} \Longrightarrow r_{H} \approx \frac{2 M}{M_{\mathrm{Pl}}^{2}}, \\
& M \approx M_{\mathrm{Pl}} \Longrightarrow r_{H} \approx \frac{2+\beta}{M_{\mathrm{Pl}}}, \\
& M \ll M_{\mathrm{Pl}} \Longrightarrow r_{H} \approx \frac{\beta}{M}
\end{aligned}
$$

for super-Planckian, Planckian, and sub-Planckian mass black holes. The horizon behaviour as a function of black hole mass is shown in Figure 1.

In [4] it is shown how though the singularity is not removed it can never be reached (it is possible that the persistence of the singularity behind such a "screen" can be related to holography and a new approach to the information loss problem; if one considers the "screen" to be a true horizon, it is also possible that this has strong implications for cosmic censorship, in that GUP black holes can never admit naked singularities). If $\beta>0$, this is done by inverting (22) in terms of the two masses $M_{ \pm}$associated with a given horizon radius $r_{H}$, which give a minimum radius

$$
r_{\min }=2 \sqrt{2 \beta} \ell_{p}
$$

and an associated mass

$$
M\left(r_{\min }\right)=\sqrt{\frac{\beta}{2}} M_{\mathrm{Pl}} .
$$

If $r_{H} \geq r_{\min }$, one recovers a gravitational ultraviolet selfcompleteness, in that the singularity can never be externally probed.

The case of $\beta<0$ is somewhat unclear in this circumstance. Negative values of the GUP parameter were originally noted in the context of lattice approach to the GUP [32] and have more recently been explored in $[33,34]$. As discussed in [4], it is only $M_{+}$solution that applies in the inverted mass relations, yielding a constrained horizon solution for $M>\sqrt{\beta / 2} M_{\mathrm{Pl}}$ from (22). It should be noted, however, that the ultraviolet cut-off is not applicable to solutions with $\beta<0$, and one finds $M \rightarrow \sqrt{|\beta| / 2} M_{\mathrm{Pl}}$, but a divergent temperature $T \rightarrow \infty$ for $r_{H} \rightarrow 0$. Thus, although solutions are possible in $\beta<0$ regime, the thermodynamic pathologies of the standard Schwarzschild solution persist.

\section{HWF of GUP Black Holes}

As previously done in [17-20,35], we can describe a massive particle at rest in the origin of a reference frame with the spherically symmetric Gaussian wavefunction

$$
\psi_{S}(r)=\frac{e^{-r^{2} / 2 \ell^{2}}}{(\ell \sqrt{\pi})^{3 / 2}}
$$

Inspired by the dual role of $m$ in the GUP, we now explore the existence of sub-Planckian black holes, that is, quantum mechanical objects that are simultaneously elementary particles and black holes. In this context, we replace our usual mass $m$ by the "GUP" mass $M$ :

$$
M \equiv m\left(1+\frac{\beta}{2} \frac{M_{\mathrm{Pl}}^{2}}{m^{2}}\right) .
$$

Next, we consider the particular case where the width $\ell$, related to the uncertainty in the size of the particle, is approximately given by the Compton length:

$$
\ell=\lambda_{m} \simeq \ell_{p} \frac{M_{\mathrm{Pl}}}{M}
$$


Noting that because the analysis holds for independent $\ell$ and $m$, such case corresponds to maximum localization for the source as one expects $\ell \geqslant \lambda_{m}$.

Taking the Fourier Transform, the corresponding wavefunction in momentum space gives

$$
\widetilde{\psi}_{S}(p)=\frac{e^{-p^{2} / 2 \Delta^{2}}}{(\Delta \sqrt{\pi})^{3 / 2}}
$$

where $\Delta=M_{\mathrm{Pl}} \ell_{p} / \ell$ is the spread of the wave-packet in momenta space.

Assuming the relativistic mass-shell equation in flat space-time to account for high-energy particle collisions, we relate the momentum $p$ to the total energy $E$ :

$$
E^{2}=p^{2}+M^{2}
$$

From the relation for the Schwarzschild radius (5) and fixing the normalization by means of (11), we then obtain the HWF:

$$
\psi_{H}\left(r_{H}\right)=\frac{1}{4 \ell_{p}^{3}} \sqrt{\frac{\ell^{3}}{\pi \Gamma(3 / 2,1)}} \Theta\left(r_{H}-R_{H}\right) e^{-\ell^{2} r_{H}^{2} / 8 \ell^{4}},
$$

where we defined $R_{H}=2 \ell_{p} M / M_{\mathrm{Pl}}$ and the Heaviside step function arises from the fact that $E \geqslant M$. Finally,

$$
\Gamma(s, x)=\int_{x}^{\infty} t^{s-1} e^{-t} d t
$$

is the upper incomplete Gamma function.

One can calculate the probability density to be

$$
P_{<}=\frac{\ell^{3}}{2 \sqrt{\pi} \ell_{p}^{6}} \frac{\gamma\left(3 / 2, r_{H}^{2} / \ell^{2}\right)}{\Gamma(3 / 2,1)} \Theta\left(r_{H}-R_{H}\right) e^{-\ell^{2} r_{H}^{2} / 4 \ell^{4}} r_{H}^{2},
$$

where $\gamma(s, x)=\Gamma(s)-\Gamma(s, x)$ is the lower incomplete Gamma function.

Integrating the density for $r_{H}$ from $R_{H}$ to infinity gives us the probability of a particle to be a black hole in terms of its mass:

$$
\begin{aligned}
\mathscr{P}_{\mathrm{BH}}(m)= & -\frac{2 \sqrt{\pi} T\left(2 \sqrt{2} m^{2}\left(\beta / 2 m^{2}+1\right)^{2}, 1 / 2 m^{2}\left(\beta / 2 m^{2}+1\right)^{2}\right)}{\Gamma(3 / 2,1)}+\operatorname{erf}\left(2 m^{2}\left(\frac{\beta}{2 m^{2}}+1\right)^{2}\right) \\
& +\frac{\sqrt{\pi} \operatorname{erfc}\left(2 m^{2}\left(\beta / 2 m^{2}+1\right)^{2}\right)}{2 \Gamma(3 / 2,1)}-\frac{2 m^{2} e^{-4 m^{4}\left(\beta / 2 m^{2}+1\right)^{4}-1}\left(4 m^{4}\left(\beta / 2 m^{2}+1\right)^{4}+3\right)\left(\beta / 2 m^{2}+1\right)^{2}}{\sqrt{\pi} \Gamma(3 / 2,1)\left(4 m^{4}\left(\beta / 2 m^{2}+1\right)^{4}+1\right)^{2}},
\end{aligned}
$$

where $T(h, a)=(1 / 2 \pi) \int_{0}^{a}\left(e^{-(1 / 2) h^{2}\left(1+x^{2}\right)} /\left(1+x^{2}\right)\right) d x$ is Owen's function.

The associated probabilities are shown in Figure 2 for positive $\beta$. We restrict this scenario to the cut-off mass shown before to correspond to the minimum radius $r_{\min }$ given by $M\left(r_{\text {min }}\right)=\sqrt{\beta / 2} M_{\mathrm{Pl}}$; and masses below this cutoff are evaluated for numerical purposes only. Nevertheless, we remind the reader that in the original GUP formulation [4] there is no minimum mass restriction; therefore the limit $M \rightarrow 0$ can theoretically be reached. First, we note that for vanishing $\beta$ the results for the probability of an elementary particle to be a black hole resemble that of the standard Schwarzschild metric [14]. This is in agreement with our modified theory, given that, for $\beta=0, M \rightarrow m$ and the horizon radius becomes the classical Schwarzschild radius.

On the other hand, for increasing $\beta$, the graph displays a minimum in the probability, meaning that for every value of the mass there is a certain chance that the particle will be a black hole. Furthermore, for sufficiently large $\beta$, everything is a black hole, in agreement with the fact the GUP imposes no minimum mass and that the effect of increasing this free parameter translates to having a bigger mass confined in a bigger horizon radius, thus making it more probable for a particle to be a black hole. In analogy with the Heisenberg Uncertainty Principle (HUP), the dashed regime of the graphs for $m \rightarrow 0$ indicates that $\Delta p \rightarrow 0$ so $\Delta x \rightarrow \infty$ like the case of a free particle in quantum mechanics; hence $\mathscr{P}_{\mathrm{BH}} \simeq 1$ making it a certainty that the particle will lie somewhere within its horizon radius and thus be a black hole.

By taking the limit $R_{H} \rightarrow 0$ in the Heaviside function, we obtain a simple analytic approximation for the HWF:

$$
\psi_{H}\left(r_{H}\right)=\left(\frac{\ell}{2 \sqrt{\pi} \ell_{p}^{2}}\right)^{3 / 2} e^{-\ell^{2} r_{H}^{2} / 8 \ell^{4}}
$$

from which, by the same procedure, one can derive the approximate probability in terms of the mass to be 


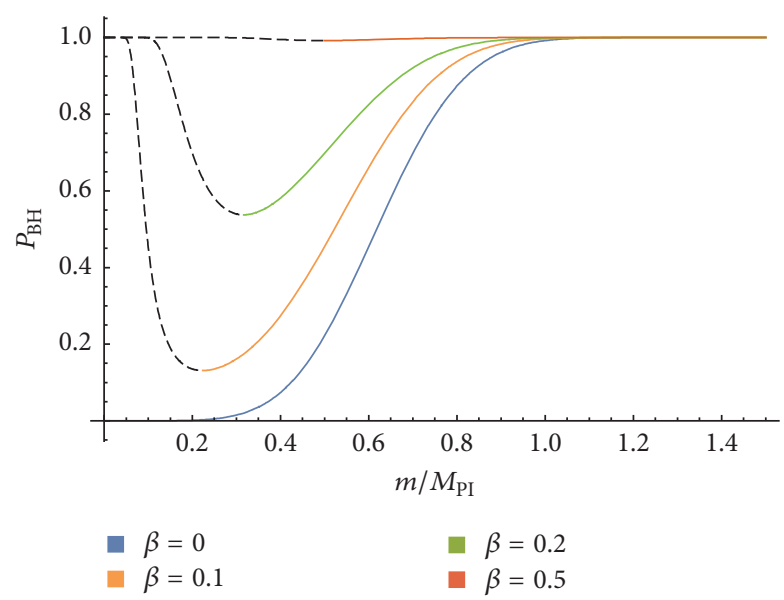

FIgURe 2: Probability $P_{\mathrm{BH}}(m)$ for a particle to be a black hole for increasing values of the free parameter $\beta$ versus mass of the particle. Dashed lines correspond to values where $m / M_{\mathrm{Pl}}<\sqrt{\beta / 2} M_{\mathrm{Pl}}$.

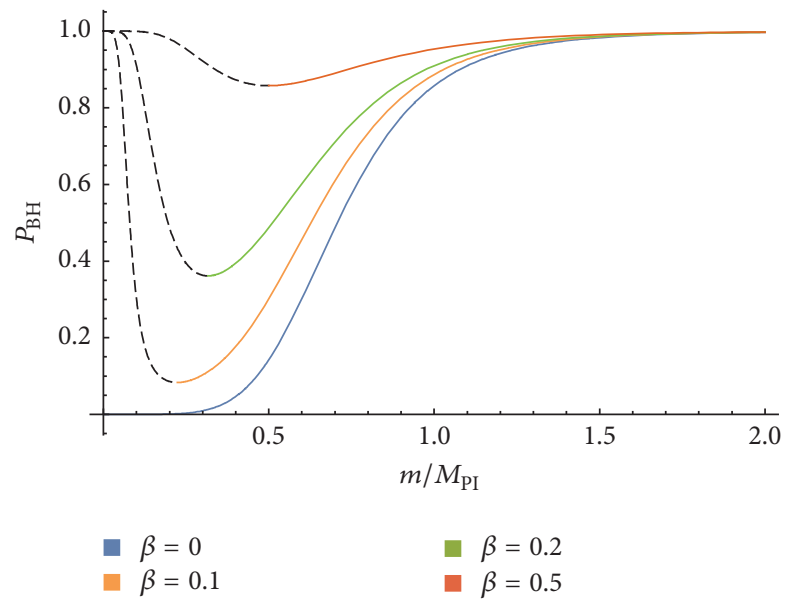

Figure 3: Analytic approximation of the probability $P_{\mathrm{BH}}(m)$ for a particle to be a black hole versus mass of the particle for increasing values of the free parameter $\beta$. Dashed lines correspond to values where $m<\sqrt{\beta / 2} M_{\mathrm{Pl}}$.

$$
\begin{aligned}
P_{\mathrm{BH}}(m) & =\left(\frac{2 m \cot ^{-1}\left(2 m^{2} /\left(\beta+2 m^{2}\right)^{2}\right)}{\beta+2 m^{2}}\right. \\
& \left.-\frac{2\left(8 m^{3} /\left(\beta+2 m^{2}\right)^{3}-2 \beta / m-4 m\right)}{\left(4 \beta+\beta^{2} / m^{2}+4 m^{2}\left(1 /\left(\beta+2 m^{2}\right)^{2}+1\right)\right)^{2}}\right) \\
& \cdot\left(\pi \sqrt{\frac{m^{2}}{\left(\beta+2 m^{2}\right)^{2}}}\right)^{-1} .
\end{aligned}
$$

Figure 3 shows graphically that this probability approximation is slight underestimate of the probability in (34).

Next, negative values of $\beta$ are considered in Figure 4. This case also presents a minimum allowed mass given by $M>\sqrt{|\beta| / 2} M_{\mathrm{Pl}}$. Nevertheless, by the time this cut-off

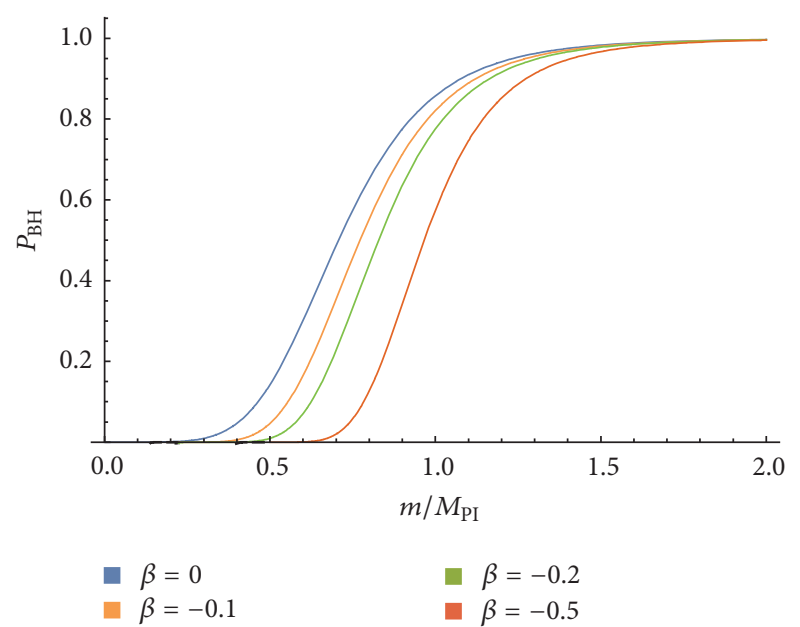

FIgURE 4: Analytic approximation of the probability $P_{\mathrm{BH}}(m)$ for a particle to be a black hole versus mass of the particle for decreasing values of the free parameter $\beta$.

comes to action $\mathscr{P}_{\mathrm{BH}} \simeq 0$ so its effect goes unnoticed in the graphs. In this case, decreasing $\beta$ is a smooth extension of the Schwarzschild scenario that simply shifts the probability curve to the right, given that $M$ and $r_{H}$ both get smaller, thus making it more improbable for the particle to be a black hole.

An important feature in Figures 2, 3, and 4 is that $P_{\mathrm{BH}} \simeq 1$ for $m \gtrsim M_{\mathrm{Pl}}$.

Similarly, $\mathscr{P}_{\mathrm{BH}}$ can be expressed in terms of the Gaussian width assuming $\ell / \ell_{p}=M_{\mathrm{Pl}} / m$ :

$$
\begin{gathered}
P_{\mathrm{BH}}(\ell)=\left|\ell \beta+\frac{2}{\ell}\right|\left(\frac{2 \ell \cot ^{-1}\left(2 \ell^{2} /\left(\beta \ell^{2}+2\right)^{2}\right)}{\beta \ell^{2}+2}\right. \\
\left.-\frac{2\left(8 \ell^{3} /\left(\beta \ell^{2}+2\right)^{3}-2 \beta \ell-4 / \ell\right)}{\left(4 \beta+\ell^{2}\left(\beta^{2}+4 /\left(\beta \ell^{2}+2\right)^{2}\right)+4 / \ell^{2}\right)^{2}}\right)(\pi)^{-1} .
\end{gathered}
$$

In Figure 5, one can see that increasing $\beta$ induces a probability for the particle to be a black hole for larger Gaussian width values, which corresponds to a big localization, in agreement with the results of Figure 3. Furthermore, the minimum mass cut-off $M>\sqrt{|\beta| / 2} M_{\mathrm{Pl}}$ induces a maximum Gaussian width cut-off given by

$$
\ell>\sqrt{\frac{2}{\beta}} \ell_{p}
$$

so no particle can physically be localized in a width bigger than the above. This may be an indication of some phenomena occurring around this scale, which can correspond to a phase transition of the black hole into something else.

Finally, Figure 6 illustrates how decreasing $\beta$ causes the probability of the particle to be a black hole to diminish for a given Gaussian width value. Again, the negative $\beta$ scenario ends up being unaffected by the imposed cut-off as before, because $\mathscr{P}_{\mathrm{BH}} \simeq 0$ when the maximum allowed localization is reached. 


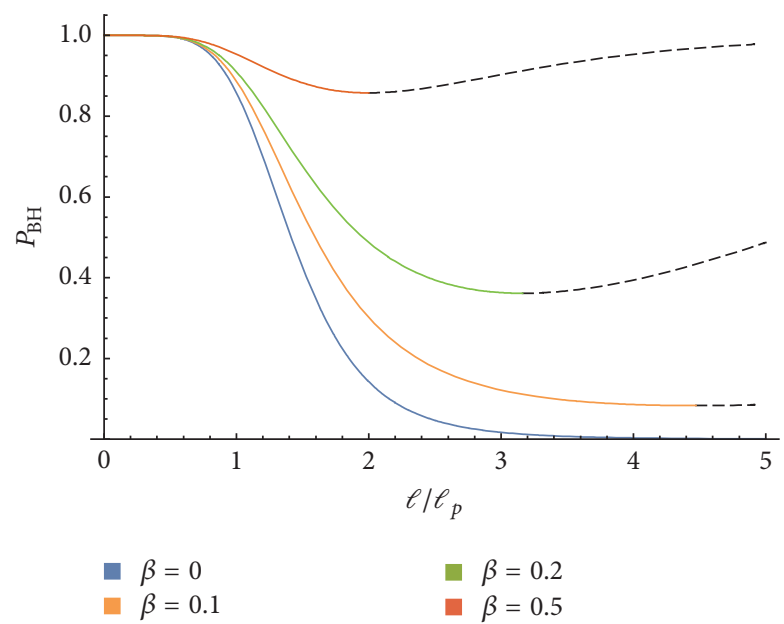

FIgURE 5: Analytic approximation of the probability $P_{\mathrm{BH}}(m)$ for a particle to be a black hole versus Gaussian width for increasing values of the free parameter $\beta$. Dashed lines correspond to the values $\ell>\sqrt{2 / \beta} \ell_{p}$.

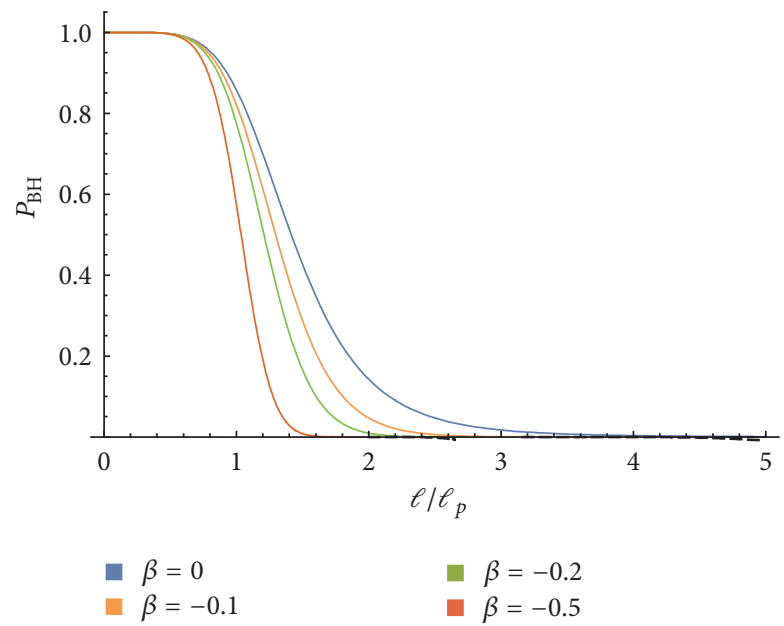

FIgURE 6: Analytic approximation of the probability $P_{\mathrm{BH}}(m)$ for a particle to be a black hole versus Gaussian width for decreasing values of the free parameter $\beta$.

It is important to emphasize in Figures 5 and 6 that $\ell \lesssim$ $\ell_{p} \rightarrow P_{\mathrm{BH}} \simeq 1$ independent of $\beta$, which is a desired intuitive result.

\section{Conclusions}

In this paper we extended the results of [13] by embedding the source in a GUP-inspired metric discussed on [4]. Considering the case of a wave-packet shaped by a Gaussian distribution, the corresponding Horizon Wavefunction was computed and the probability $\mathscr{P}_{\mathrm{BH}}$ that the source is a (quantum) black hole, that is, that it lies within its horizon radius, was calculated.

The case for $\beta<0$ looks qualitatively similar to the standard Schwarzschild case, which is recovered when $\beta=0$.
The general shape of $\mathscr{P}_{\mathrm{BH}}$ is maintained when decreasing the free parameter and shifted to reduce the probability for the particle to be a black hole accordingly. Effectively, a negative $\beta$ reduces the magnitude of $M$ and consequently $R_{H}$, thus matching our intuition that the particle should be either more localized or more massive in order to be a black hole.

$\beta>0$ scenario differs in many ways from the previous one. First of all, when increasing the free parameter a minimum in $\mathscr{P}_{\mathrm{BH}}$ is encountered, thus meaning that every particle has some probability of decaying to a black hole. Furthermore, for sufficiently large $\beta$ one gets that every particle is a quantum black hole. Again, these results are in agreement with the intuitive effect of increasing $\beta$, which creates larger $M$ and $R_{H}$ terms, thus making it more probable for the particle to lie within horizon radius and hence be a black hole.

To conclude, although in our approach we restrict the sub-Planckian regime by imposing a mass/localization cutoff, the exact nature of extremely low mass black holes ultimately depends on whatever the correct model of quantum gravity is. It might be the case that this is something described by, for example, the quantum $N$ portrait approach for low graviton number [36-38].

Finally, possible ways of extending this investigation include introducing the GUP formulation of HQM by modifying the momentum-space wavefunction with one that encodes the generalized uncertainty relation:

$$
\psi_{\xi}^{m l}(p)=\sqrt{\frac{2 \sqrt{\beta}}{\pi}}\left(1+\beta p^{2}\right)^{-1 / 2} e^{-i\left(\xi \tan ^{-1}(\sqrt{\beta} p) / \hbar \sqrt{\beta}\right)}
$$

as proposed in [29]. Furthermore, future research can analyze the effect of modifying the Heaviside function in (34) to one where the edges are smoothed by the "golden rule" [39] because of the uncertainty introduced at the quantum scale.

\section{Competing Interests}

The authors declare that they have no competing interests.

\section{Acknowledgments}

Luciano Manfredi and Jonas Mureika thank Roberto Casadio and Piero Nicolini for insightful discussions on the manuscript. Luciano Manfredi was supported by a Frank R. Seaver Summer Undergraduate Research Scholarship from Loyola Marymount University.

\section{References}

[1] T. Johannsen, A. E. Broderick, P. M. Plewa et al., "Testing general relativity with the shadow size of Sgr A*," Physical Review Letters, vol. 116, no. 3, Article ID 031101, 2016.

[2] P. Nicolini, A. Smailagic, and E. Spallucci, "Noncommutative geometry inspired Schwarzschild black hole," Physics Letters B, vol. 632, no. 4, pp. 547-551, 2006.

[3] P. Nicolini, "Noncommutative black holes, the final appeal to quantum gravity: a review," International Journal of Modern Physics A, vol. 24, no. 7, p. 1229, 2009. 
[4] B. Carr, J. Mureika, and P. Nicolini, "Sub-Planckian black holes and the generalized uncertainty principle," Journal of High Energy Physics, 2015.

[5] B. J. Carr, "The black hole uncertainty principle correspondence," in Proceedings of the 1st Karl Schwarzschild Meeting on Gravitational Physics, vol. 170 of Springer Proceedings in Physics, pp. 159-167, 2015.

[6] M. Reuter and J. M. Schwindt, "A minimal length from the cutoff modes in asymptotically safe quantum gravity," Journal of High Energy Physics, vol. 2006, no. 1, p. 70, 2006.

[7] R. Ruffini and S. Bonazzola, "Systems of self-gravitating particles in general relativity and the concept of an equation of state," Physical Review, vol. 187, no. 5, pp. 1767-1783, 1969.

[8] M. Bahrami, A. Großardt, S. Donadi, and A. Bassi, "The Schrödinger-Newton equation and its foundations," New Journal of Physics, vol. 16, no. 11, 2014.

[9] A. Großardt, J. Bateman, H. Ulbricht, and A. Bassi, "Optomechanical test of the Schrödinger-Newton equation," Physical Review D, vol. 93, no. 9, Article ID 096003, 6 pages, 2016.

[10] P. C. W. Davies, "Quantum field theory in curved space-time," Nature, vol. 263, no. 5576, pp. 377-380, 1976.

[11] N. D. Birrell and P. C. Davies, Quantum Fields in Curved Space, vol. 7 of Cambridge Monographs on Mathematical Physics, Cambridge University Press, Cambridge, UK, 1982.

[12] T. Jacobson, "Introduction to Quantum Fields in Curved Spacetime and the Hawking Effect," https://arxiv.org/abs/gr-qc/ 0308048.

[13] R. Casadio, "Localised particles and fuzzy horizons: A tool for probing Quantum Black Holes," https://arxiv.org/abs/1305.3195.

[14] R. Casadio, A. Giugno, and O. Micu, "Horizon quantum mechanics: a hitchhiker's guide to quantum black holes," International Journal of Modern Physics. D. Gravitation, Astrophysics, Cosmology, vol. 25, no. 2, Article ID 1630006, 45 pages, 2016.

[15] R. Casadio, A. Giugno, O. Micu, and A. Orlandi, "Thermal BEC black holes," Entropy, vol. 17, no. 10, pp. 6893-6924, 2015.

[16] X. Calmet and R. Casadio, "The horizon of the lightest black hole," The European Physical Journal C, vol. 75, no. 9, article no. 445, 2015.

[17] R. Casadio, "What is the Schwarzschild radius of a quantum mechanical particle?" Springer Proceedings in Physics, vol. 170, pp. 225-231, 2016.

[18] R. Casadio and F. Scardigli, "Horizon wave function for single localized particles: GUP and quantum black-hole decay," The European Physical Journal C, vol. 74, article no. 2685, 2014.

[19] R. Casadio, O. Micu, and F. Scardigli, "Quantum hoop conjecture: black hole formation by particle collisions," Physics Letters. $B$, vol. 732, pp. 105-109, 2014.

[20] R. Casadio, R. T. Cavalcanti, A. Giugno, and J. Mureika, "Horizon of quantum black holes in various dimensions," Physics Letters B, vol. 760, pp. 36-44, 2016.

[21] N. Arsene, R. Casadio, and O. Micu, "Quantum production of black holes at colliders," The European Physical Journal C, vol. 76, no. 7, article no. 384, 2016.

[22] G. Veneziano, "A stringy nature needs just two constants," Europhysics Letters, vol. 2, no. 3, pp. 199-204, 1986.

[23] A. Ashtekar, S. Fairhurst, and J. L. Willis, "Quantum gravity, shadow states and quantum mechanics," Classical and Quantum Gravity, vol. 20, no. 6, pp. 1031-1061, 2003.

[24] S. Majid, "Hopf algebras for physics at the Planck scale," Classical and Quantum Gravity, vol. 5, no. 12, 1988.
[25] M. Isi, J. Mureika, and P. Nicolini, "Self-completeness and the generalized uncertainty principle," Journal of High Energy Physics, no. 11, 2013.

[26] M. Maggiore, "The algebraic structure of the generalized uncertainty principle," Physics Letters. B, vol. 319, no. 1-3, pp. 83-86, 1993.

[27] M. Maggiore, "Quantum groups, gravity, and the generalized uncertainty principle," Physical Review. D. Third Series, vol. 49, no. 10, pp. 5182-5187, 1994.

[28] K. S. Thorne, "Nonspherical gravitational collapse: a short review," in Magic without Magic, J. R. Klauder, Ed., pp. 231-258, W. H. Freemann, San Francisco, Calif, USA, 1972.

[29] A. Kempf, G. Mangano, and R. B. Mann, "Hilbert space representation of the minimal length uncertainty relation," Physical Review. D. Third Series, vol. 52, no. 2, pp. 1108-1118, 1995.

[30] F. Scardigli, "Generalized uncertainty principle in quantum gravity from micro-black hole gedanken experiment," Physics Letters B, vol. 452, no. 1-2, pp. 39-44, 1999.

[31] R. J. Adler and D. I. Santiago, "On gravity and the uncertainty principle," Modern Physics Letters A, vol. 14, no. 20, pp. 13711381, 1999.

[32] P. Jizba, H. Kleinert, and F. Scardigli, "Uncertainty relation on a world crystal and its applications to micro black holes," Physical Review D, vol. 81, no. 8, 2010.

[33] F. Scardigli and R. Casadio, "Gravitational tests of the generalized uncertainty principle," The European Physical Journal C, vol. 75, no. 9, p. 425, 2015.

[34] A. Farag Ali, M. M. Khalil, and E. C. Vagenas, "Minimal length in quantum gravity and gravitational measurements," EPL (Europhysics Letters), vol. 112, no. 2, 2015.

[35] R. Casadio, A. Giugno, and A. Giusti, "Matter and gravitons in the gravitational collapse," Physics Letters B, vol. 763, pp. 337340, 2016.

[36] G. Dvali, C. Gomez, and D. Lüsta, "Classical limit of black hole quantum $N$-portrait and BMS symmetry," Physics Letters B, vol. 753, pp. 173-177, 2016.

[37] G. Dvali and C. Gomez, "Black hole macro-quantumness," https://arxiv.org/abs/1212.0765.

[38] A. Frassino, S. Köppel, and P. Nicolini, "Geometric model of black hole quantum $\mathrm{N}$-portrait, extradimensions and thermodynamics," Entropy, vol. 18, no. 5, article no. 181, 2016.

[39] J. Mureika, P. Nicolini, and E. Spallucci, "Could any black holes be produced at the LHC?” Physical Review D, vol. 85, no. 10, 2012. 

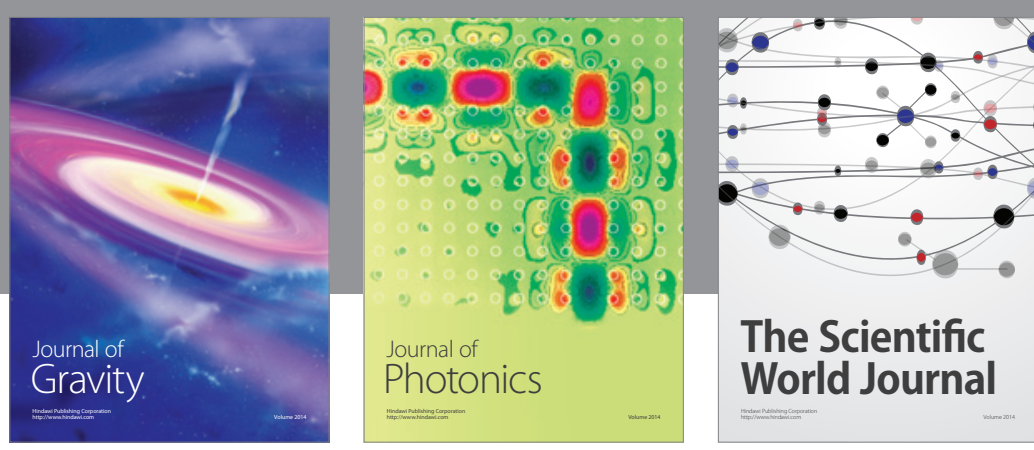

The Scientific World Journal
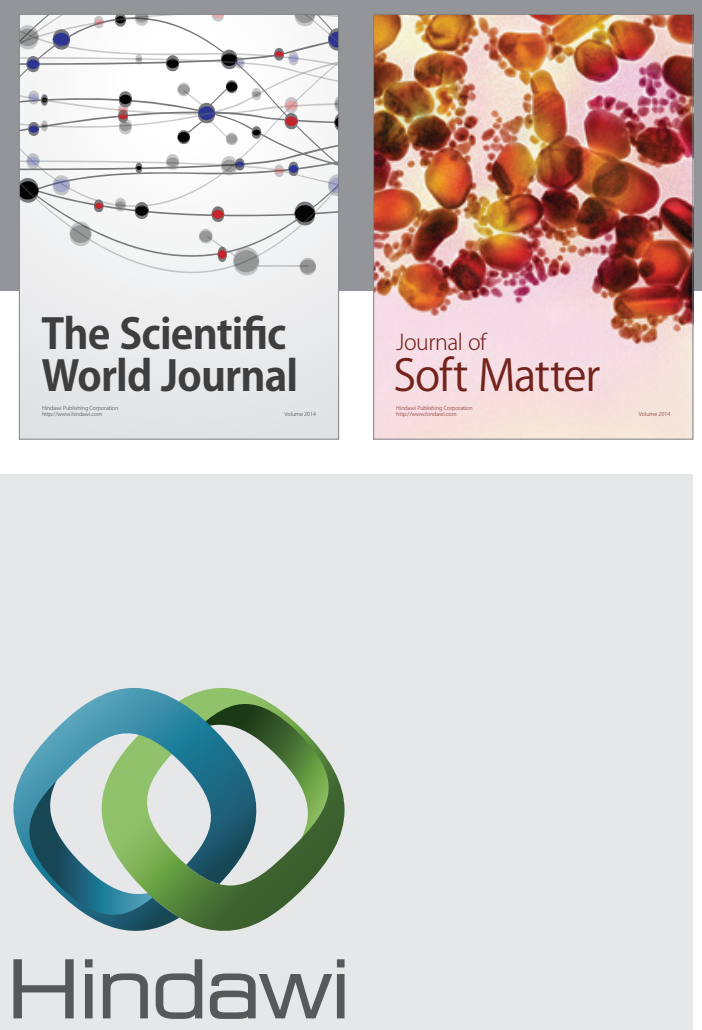

Submit your manuscripts at

http://www.hindawi.com

nternational Journal of

Statistical Mechanics
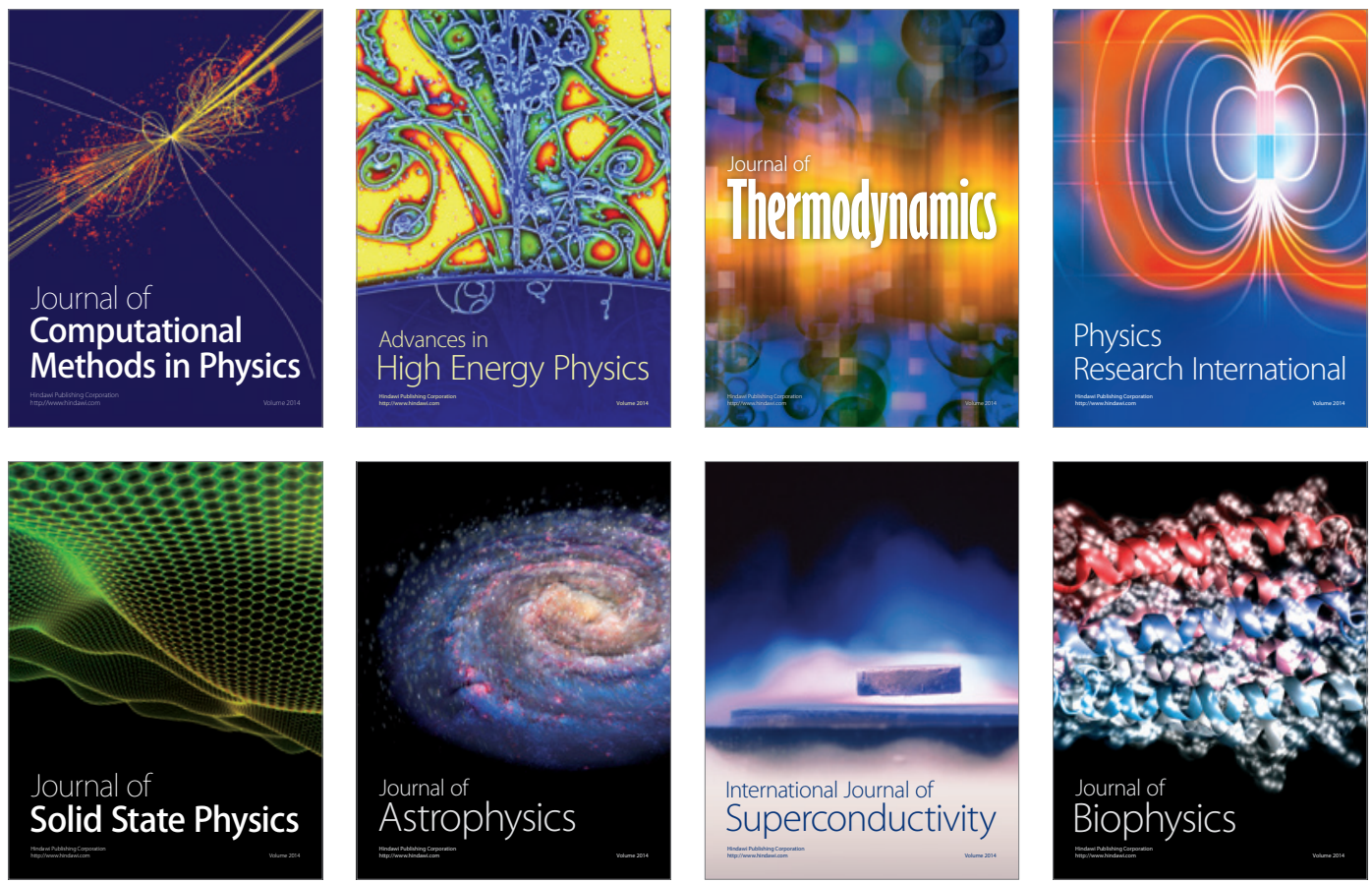
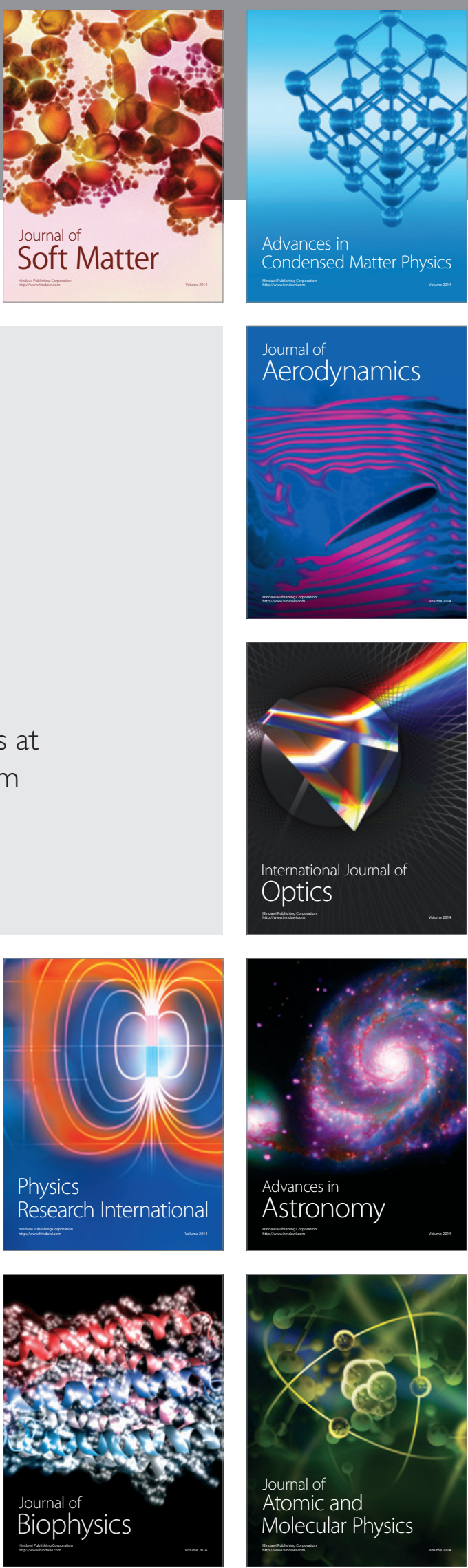